\title{
Mechanisms of Lipotoxicity in the Cardiovascular System
}

\author{
Adam R. Wende $^{1}$, J. David Symons ${ }^{1,2}$, and E. Dale Abel ${ }^{1}$ \\ ${ }^{1}$ Program in Molecular Medicine and Division of Endocrinology, Metabolism, and Diabetes, \\ University of Utah, School of Medicine, Salt Lake City, UT 84112 \\ ${ }^{2}$ College of Health, University of Utah, School of Medicine, Salt Lake City, UT 84112
}

\section{Abstract}

Cardiovascular diseases account for approximately one third of all deaths globally. Obese and diabetic patients have a high likelihood of dying from complications associated with cardiovascular dysfunction. Obesity and diabetes increase circulating lipids that upon tissue uptake, may be stored as triglyceride, or may be metabolized in other pathways, leading to the generation of toxic intermediates. Excess lipid utilization or activation of signaling pathways by lipid metabolites may disrupt cellular homeostasis and contribute to cell death, defining the concept of lipotoxicity. Lipotoxicity occurs in multiple organs, including cardiac and vascular tissues, and a number of specific mechanisms have been proposed to explain lipotoxic tissue injury. In addition, recent data suggests that increased tissue lipids may also be protective in certain contexts. This review will highlight recent progress toward elucidating the relationship between nutrient oversupply, lipotoxicity, and cardiovascular dysfunction. The review will focus in two sections on the vasculature and cardiomyocytes respectively.

\section{Keywords}

Heart; Vasculature; Nutrient oversupply; Lipotoxicity; Pathways and mechanisms; Cardiac function; Vascular dysfunction; Cardiovascular disease; Ceramide; Adiponectin; Nitric oxide; Reactive oxygen species; MicroRNA; Epigenetics; Autophagy; Insulin resistance; Obesity; Inflammation; Mitochondria; Endothelial dysfunction; Glucotoxicity; Cardiac lipoprotection; Diabetes; Hypertension

\section{Introduction}

Cardiovascular complications are the leading causes of morbidity and mortality in individuals with diet-induced obesity (DIO), insulin resistance, and type 2 diabetes mellitus (T2DM). Complications include pathologies specific to large vessels (atherosclerosis, cardiomyopathy) and small vessels (retinopathy, nephropathy, neuropathy). There are multiple mechanisms that contribute to these clinical outcomes, including hyperglycemia, hyperinsulinemia, insulin resistance, inflammation, changes in circulating concentrations of adipokines, and alterations in intracellular signaling pathways. Increased circulating concentrations of lipids and altered tissue metabolism of lipids are consistent features of these prevalent conditions and contribute importantly to cardiovascular complications. Impairments in tissue homeostasis that is attributable to alterations in lipid utilization, or

Address correspondence to: E. Dale Abel, MD, PhD, Division of Endocrinology, Metabolism, and Diabetes, and Program in Molecular Medicine, 15 N 2030 E., Bldg. \#533, Rm. 3110B, Salt Lake City, Utah 84112, Phone: (801) 585-0727 Fax: (801) 585-0701 dale.abel@hmbg.utah.edu.

A.R. Wende and J.D. Symons contributed equally to this work.

Disclosure No potential conflicts of interest relevant to this article were reported. 
lipid-induced changes in intracellular signaling, have been broadly termed lipotoxicity. This review will focus on the mechanisms linking increased tissue lipid uptake, metabolism, and utilization with vascular dysfunction and changes in cardiac contractility. We will not focus on atherosclerosis, which has been the subject of many excellent reviews.

\section{Part 1. Lipotoxicity and Vascular Dysfunction}

Endothelial dysfunction is a hallmark of obesity and diabetes-related vascular dysfunction. A central aspect of endothelial dysfunction is reduced nitric oxide (NO) bioavailability. Multiple alterations in the metabolic milieu, such as hyperglycemia; oxidative stress; activation of the renin-angiotensin system and increased pro-inflammatory cytokines that are present in individuals with diet-induced obesity (DIO); type 2 diabetes mellitus (T2DM); and insulin resistance, independently and synergistically contribute to decreasing NO bioavailability. This section will focus on specific mechanisms that are attributable to lipotoxicity. We will review key mechanisms by which lipotoxicity may decrease endothelial NO synthase (eNOS) gene expression and eNOS catalytic activity, leading to endothelial cell dysfunction. These include vascular insulin resistance, inflammation, oxidative stress, accumulation of toxic lipid metabolites, and mitochondrial dysfunction.

\section{Insulin Resistance in Endothelial Cells}

It is generally accepted that vascular insulin resistance correlates with endothelial dysfunction [1]. This relationship is driven by a pathway-selective inhibition of insulinmediated activation of eNOS by PI3K and Akt, whereas MAPK signaling to endothelin 1

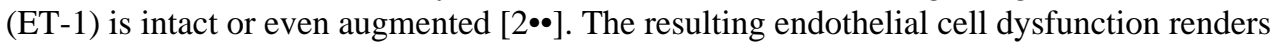
the vascular wall more susceptible to atherosclerosis and less responsive to agonist-induced vasodilation $[3 \bullet, 4 \cdot]$.

Excessive free fatty acids (FFAs) can promote de novo synthesis of diacylglycerol that activates classic and novel protein kinase $\mathrm{C}(\mathrm{PKC})$ isoforms [5, 6]. PKC activation inhibits insulin and Vascular endothelial growth factor (VEGF)-mediated activation of PI3K and Akt $[7,2 \bullet \bullet]$, which limits cGMP formation from nitric oxide (eNOS generated) activation of guanylate cyclase [8•]. An examination of multiple targets in the insulin-signaling pathway in endothelial cells revealed that general (phorbol ester) and specific (angiotensin II) PKC activation [4•] (particularly the PKCa isoform) phosphorylates a novel site (Thr-86) on the p85a subunit of PI3K. Phosphorylation of Thr-86 reduces the binding of p85a to insulin receptor (IR) substrate 1 (IRS1), and decreases insulin and VEGF signaling via PI3K to eNOS. Whole-body IRS1 knockout (KO) mice are hypertensive and endothelial specific IRS1 KO mice display endothelial dysfunction [9], supporting the functional importance of intact IRS1-mediated PI3K signaling to eNOS.

IR substrate 2 (IRS2) also plays an important role in relaying the insulin signal to eNOS. Endothelial cell IRS2 expression, Akt activation, and p-eNOS are decreased and capillary recruitment and insulin delivery are impaired in fat-fed vs. lean mice [10••]. These defects are neutralized when fat-fed mice are treated with a prostacyclin analog that increases eNOS expression, and all findings are recapitulated in endothelial-specific IRS2 KO mice. Taken together, these observations provide strong evidence linking obesity and its associated increase in circulating FFAs to impaired insulin-mediated signaling to eNOS in endothelial cells to an extent that could be physiologically relevant.

Other genetic models have been used to demonstrate important functional consequences of disrupted IR-mediated signaling to eNOS. Mice with germ line haploinsufficiency of the IR $\left(\mathrm{IR}^{+/-}\right.$mice) display hypertension, mild insulin resistance, reduced basal and insulinstimulated eNOS phosphorylation in the vasculature, and an age-dependent decrease in 
arterial vasorelaxation that is associated with an increase in endothelial cell-derived NADPH oxidase-mediated $\mathrm{O}_{2}{ }^{\bullet-}$ production $[11 \bullet, 12]$. Endothelial regeneration in response to wireinduced denudation of the femoral artery is delayed in $\mathrm{IR}^{+/-}$vs. wild-type (WT) mice, but this component of the $\mathrm{IR}^{+/-}$phenotype was rescued by transfusion of angiogenic progenitor cells obtained from insulin-sensitive, but not from insulin-resistant, animals [13••]. In a murine model wherein apolipoprotein (apo) E-deficient mice are crossed to mice with IR deletion in endothelial cells, atherosclerotic lesion size, endothelium-dependent dysfunction, and VCAM-1 expression are most severe in double-KO mice [3•]. Thus, defective IR signaling in the vascular endothelium, in the absence of changes in systemic metabolism, promotes early events in atherogenesis and accelerates the progression of atherosclerotic disease. However, not all studies in genetic models of IR disruption support the hypothesis that vascular insulin resistance is sufficient to induce vascular dysfunction. IR-null mice with transgenic re-expression of the IR in brain, liver, and pancreatic $\beta$-cells $\left(T T r-I R^{-/-}\right.$ mice) exhibit preserved glucose homeostasis that is associated with hyperinsulinemia [14]. In these mice, no differences in blood pressure or in the gene expression levels of ET-1 or eNOS, or in eNOS phosphorylation, are observed between groups, despite a complete loss of insulin-stimulated activation of intracellular signaling kinases. Furthermore, endotheliumdependent vasorelaxation and indices of oxidant stress are unchanged in vessels from $T T_{T}$ $I R^{-/-}$vs. WT controls [15••]. These observations suggest complex interactions between IR haploinsufficiency, loss of IR or IRS isoforms, and the presence or absence of hyperinsulinemia in the vascular adaptation to genetic perturbation of insulin signaling.

\section{Cross Talk Between IR and Insulin-Like Growth Factor 1 Receptors (IGF-1Rs)}

-Insulin-like growth factor 1 receptors (IGF-1Rs) exist in the vasculature and heterodimerize with IRs to form hybrid receptors in insulin resistant conditions associated with DIO and T2DM. Because these hybrid receptors bind to IGF-1 but not insulin with high affinity, they may attenuate insulin-mediated NO production by the endothelium. Thus, arteries from mice with germ line haploinsufficiency of the IGF-1R (IGF-1R ${ }^{+/-}$mice) and endothelium-specific homozygous or heterozygous disruption of the IGF-1R display blunted phenylephrine-induced vasocontraction, increased basal NO production, and enhanced insulin-mediated NO production [16•], which are reversed in mice with double $\mathrm{KO}$ of $\mathrm{IR}^{+/-}$ and IGF-1R ${ }^{+/-}[16 \bullet]$. These data suggest that modulation of IR/IGF-1R interactions could be a potential therapeutic target for ameliorating vascular dysfunction that is associated with insulin-resistance.

\section{Insulin Resistance in Intact Arteries}

Jiang et al. first reported that selective resistance via the IR-PI3K-Akt pathway exists in vessels from obese vs. lean rats $[2 \bullet \bullet]$. This issue has been examined more recently in aortae from lean and fat-fed mice $[17 \bullet, 18-20,15 \cdot \bullet]$. When directly examined, the ability of insulin to stimulate Akt phosphorylation was variable, ranging from full activation of Akt phosphorylation to modest inhibition of Akt phosphorylation following insulin stimulation. Despite the variability in Akt phosphorylation, all of these studies revealed striking suppression both in basal and insulin-stimulated eNOS phosphorylation. Functional consequences of reduced vascular NO bioavailability in obese mice observed in those studies include arterial inflammation [17•, 18, 20], impaired arterial vasorelaxation [19, $15 \bullet \cdot$, and hypertension [15••]. These data provide evidence that a dissociation may exist between insulin-mediated signaling and Akt in the vasculature, and impaired eNOS enzyme function in insulin-resistant conditions associated with DIO and T2DM [17•, 18-20, 15••].

We reasoned that additional systemic metabolic changes could account for the profound failure in insulin-mediated eNOS phosphorylation in arteries from obese vs. lean mice, despite intact signaling in the vasculature via Akt. Because fasting hyperglycemia was mild 
in fat-fed vs. lean mice, and $\mathrm{O}_{2}{ }^{--}$, NADPH-oxidase activity, AMP-dependent kinase (AMPK), and PKA are similar in vessels from lean and obese mice in our study [15••], we explored whether elevated FFAs in general, or a toxic metabolite of FFA such as the sphingolipid ceramide [21,22], could contribute to these changes. When vascular ceramide biosynthesis was prevented in fat-fed mice using pharmacological and genetic approaches, impaired eNOS phosphorylation, endothelial dysfunction, and systemic hypertension were prevented [23••]. To determine molecular mechanisms whereby ceramide might exert its effects, bovine aortic endothelial cells (BAECs) are incubated with palmitate. Palmitate increases de novo ceramide synthesis, which reduces agonist-stimulated (e.g., insulin and vascular endothelial cell growth factor) eNOS phosphorylation at S1177 and S617, eNOS dimer formation, and NO production in a ceramide-dependent manner [23・•]. In contrast to findings of others using different cell lines, the changes we observed are not due to impaired upstream signaling to eNOS from Akt, AMPK, or ERK 1/2, or to $\mathrm{O}_{2}{ }^{--}$-mediated peroxynitrite formation. Instead, ceramide induces colocalization of the protein phosphatase 2A (PP2A) with eNOS, prevents eNOS from associating with Akt and Hsp90, decreased the phosphorylation of the pool of Akt that associates directly with eNOS, and impairs full eNOS phosphorylation. Ceramide might initiate PP2A association with eNOS by disrupting the interaction between inhibitor 2 of PP2A (I2PP2A) and PP2A. Collectively, these results identify an important role for endogenous ceramide accumulation in the pathogenesis of vascular dysfunction, and extended earlier observations, implicating impaired AMPK phosphorylation in ceramide-mediated vascular dysfunction [24].

\section{Inflammation}

Palmitic, oleic, and linoleic acids are abundant fatty acids in human serum that comprise $70 \%$ of the total circulating FFAs [25]. Palmitate signals via the toll-like receptor 4 (TLR4), a pattern recognition receptor that is essential for initiating inflammatory responses associated with innate immunity [26,27]. When palmitate signals via TLR4, inhibitor of $\kappa \mathrm{B}$-kinase (IKK) is activated, which phosphorylates and degrades I $\mathrm{Ba}$, allowing nuclear translocation of NF- $\kappa \beta$, a transcriptional activator of the expression of many genes involved in inflammation [26]. The subsequent increase in the expression and synthesis of inflammatory mediators such as IL-6, TNFa, ICAM, and PAI-1 are associated with decreased endothelial NO production [28•, 17•, 29]. Increased p-IкBa, IL-6, and ICAM are observed in lysates of aorta from fat-fed vs. lean WT, but not TLR ${ }^{-/-}$, mice $[17 \bullet, 18]$. The Kim group subsequently demonstrated in endothelial cells and confirmed in vascular tissue from obese WT and TLR4 ${ }^{-1-}$ mice that an important link between palmitate-mediated TLR4 activation and IKK / p-I $\kappa \mathrm{Ba} / \mathrm{NF}-\kappa \beta$ signaling involves NADPH oxidase-mediated $\mathrm{O}_{2}{ }^{\circ-}$ production [30]. NOX4 is the most abundant isoform of NADPH oxidase in vascular tissue. Bone morphogenic protein 4 (BMP4) induces and activates NADPH oxidase in endothelial cells. Administration of BMP4 to mice induces arterial dysfunction and hypertension in an NADPH-oxidase dependent manner [31•]. Maloney et al. show that endothelial cells treated with palmitate and aortae from fat-fed mice display elevated BMP4 and NOX4 and indices of IKK / p-I $\kappa \mathrm{Ba} / \mathrm{NF}-\kappa \beta$ signaling in a TLR4-dependent manner. In addition to stimulating NF- $\kappa \beta$-mediated inflammatory responses, NADPH oxidase-mediated $\mathrm{O}_{2}{ }^{\bullet-}$ production may impair NO bioavailability and precipitate vascular dysfunction by additional mechanisms. When endogenous cellular antioxidants are incapable of responding adequately to the imposed oxidant load, $\mathrm{O}_{2}{ }^{--}$may combine with $\mathrm{NO}$ to form peroxynitrite, which disrupts the eNOS dimer, thereby reducing eNOS activity and arterial vasorelaxation [19, 32, 33]. Thus, the FFA palmitate signals via TLR4 to increase oxidant load and increase NF- $\kappa \beta$-mediated signaling in endothelial cells. Some studies question whether toll-like receptors directly bind saturated fatty acids [34], and others suggest that saturated fatty acids may not induce inflammation via TLR-mediated signaling [35]. For example, FFAs have been shown to amplify the inflammatory responses of monocytes / macrophages to bacterial 
lipopolysaccharide via a TLR4-independent mechanism that is dependent upon ceramide generation, which in turn activates PKC and MAPK [27]. Despite these controversies, the balance of the evidence favors the conclusion that FFA-mediated signaling may contribute to endothelial dysfunction by mechanisms that involve the activation of inflammatory pathways.

An important link between lipid accumulation, inflammation, and NO bioavailability might involve IKK-mediated ceramide biosynthesis. Holland et al. show that lard oil infusion increases skeletal muscle and liver ceramide synthesis in WT but not TLR4-null mice; and that palmitate incubation increases ceramide synthesis and transcript levels of enzymes involved in ceramide biosynthesis (Sptlc1, SptIc2, Des I) in cells with intact IKK kinase signaling, but not in IKK kinase-dead cells [36•]. To determine if these findings could be translated to the whole animal, mice were fed standard or high-fat chow for 4 months, and then a subgroup was switched to a diet supplemented with the IKK $\beta$ inhibitor sodium salicylate. Although body weights did not change, insulin and glucose tolerance were improved, and ceramide accumulation in skeletal muscle and liver was prevented. Vascular function was not determined in this study, but based on studies in myriocin-treated animals on a high-fat diet, it would be predicted that sodium salicylate would be expected to improve vascular function. Other approaches to suppressing flux through this pathway have also proved beneficial. Diosgenin, a steroidal sapogenin, reduces palmitate-induced IKK $\beta$ and NF- $\kappa \beta$ phosphorylation in endothelial cells to an extent that impaired the production of inflammatory markers and restored insulin-stimulated IRS-1 tyrosine phosphorylation [29]. Diosgenin restores insulin-stimulated NO production and endothelium-dependent vasorelaxation in arterial rings treated with palmitate, indicating the potential relevance of targeting this pathway in vascular diseases associated with a lipotoxic milieu. Collectively, palmitate signaling via toll-like receptor or related signaling pathways may induce vascular dysfunction by promoting: (i) IKK / p-I $\kappa \mathrm{Ba} / \mathrm{NF}-\mathrm{\kappa} \beta$ signaling that promotes inflammation; (ii) NADPH-oxidase-mediated $\mathrm{O}_{2}{ }^{-}$production that may uncouple eNOS function and exacerbate oxidative stress; and (iii) ceramide biosynthesis which may lead to PP2Amediated dephosphorylation of eNOS.

\section{Cross Talk Between Adiponectin and Ceramide}

In addition to its insulin-sensitizing, antiapoptotic, and anti-inflammatory functions, adiponectin appears to regulate sphingolipid metabolism, and this link might have consequences concerning arterial function. Once formed, ceramide can be hydrolyzed by ceramidases to produce sphingosine. Sphingosine kinase can then phosphorylate sphingosine to sphingosine-1-phosphate (SIP). Ceramide and sphingosine are pro-apoptotic, whereas SIP is generally regarded as pro-survival. This led to the hypothesis that the ceramide / SIP balance might be important concerning cell fate [37]. Hepatic ceramide accumulation in lipid-infused and fat-fed rodents can be normalized by administering recombinant adiponectin; prevented in mice that transgenically overexpress adiponectin; and amplified in adiponectin null mice [38••]. The adiponectin receptors AdipoR1 and AdipoR2 belong to the progesterone and adiponectin $\mathrm{Q}$ receptor (PAQR) family [39]. Because some PAQR family members enhance ceramidase activity, investigators sought to determine whether AdipoR1 and AdipoR2 might mediate the ability of adiponectin to lower ceramide. Adiponectin increases ceramidase activity in mouse embryonic fibroblasts (MEFs) with intact adiponectin receptors, but fails to do so in MEFs in which both isoforms are deleted. Consistent with lower ceramidase activity in AdipoR1-deficient and AdipoR2-deficient MEFs, there was diminished accumulation of SIP, such that the ceramide / SIP ratio was 5fold greater relative to WT cells. The disrupted ceramide-to-SIP balance increases the susceptibility of AdipoR1/2 deficient cells to palmitate-induced cell death that is prevented when these MEFs are treated with SIP. This signaling pathway might also be relevant in 
endothelial cells. For example, SIP signals through the endothelial differentiation gene-1 receptor to the heterotrimeric $\mathrm{G}$ protein $\mathrm{G}_{\mathrm{i}}$, to activate Akt and eNOS, and to increase NO production [40•]. Although this would be predicted to increase arterial vasorelaxation, in vivo studies focusing on vascular function, in which this pathway is manipulated, remain to be performed. Indirect support comes from studies in which adiponectin delivery to mice with T2DM can improve arterial vasorelaxation by increasing NO bioavailability [41].

\section{Mitochondria}

Mitochondria regulate cell survival and ion homeostasis. Endothelial mitochondria are responsible for generating reactive oxygen species and maintaining the cytosolic $\mathrm{Ca}^{2+}$ concentration. Evidence that changes in mitochondrial morphology and function correlate with endothelial cell dysfunction in the context of diabetes is emerging [42•, 43]. Increased mitochondrial fission or attenuated fusion disrupts the mitochondrial network and leads to endothelial dysfunction. Mitochondrial fission may increase reactive oxygen species generation and $\mathrm{Ca}^{2+}$ overload, which could potentially disrupt endothelial function and cell survival [44]. Palmitate, exposure but not hyperglycemia or hyperinsulinemia, induces mitochondrial fragmentation in differentiated $\mathrm{C}_{2} \mathrm{C}_{12}$ muscle cells and increases reactive oxygen species production [45]. Similar findings are observed in skeletal muscle of mice with genetic obesity and DIO. Increased expression of Fis 1 and mitochondrial fragmentation are observed in endothelial cells isolated from patients with T2DM [43]. Mitochondrialreactive oxygen species generation was increased, and agonist-stimulated NO production suppressed, in endothelial cells from diabetics that correlated with reduced flow-mediated dilation. While hyperglycemia was present in the diabetic patients in this study, triglycerides were moderately, but not significantly, elevated and FFAs were not measured. Thus, hyperglycemia correlates with mitochondrial fragmentation and endothelial cell dysfunction. It remains to be established if lipid excess per se contributes to this phenomenon.

\section{Summary, Part 1}

Endothelial dysfunction is a well-established characteristic of insulin resistance and obesity. Multiple mechanisms conspire to impair vascular function in this prevalent condition. Recent findings provide strong evidence that increased circulating lipids may impair vascular function in vivo by impairing insulin signaling, promoting ceramide accumulation, increasing inflammation and disrupting mitochondrial dynamics. It is likely that these changes are not parallel pathways, but are interrelated and synergistically interacting to promote vascular dysfunction (Fig. 1).

\section{Part 2. Lipotoxicity and Cardiac Function}

Maintenance of cardiac contractile function requires a constant energy supply through mitochondrial generation of adenosine-5'-triphosphate (ATP). In the healthy heart, the primary substrate for ATP synthesis is fatty acids. However, throughout development and in response to various stimuli, the heart exhibits metabolic flexibility to alter substrate preference and mitochondrial efficiency. A consequence of modern civilization is the relationship between nutrient excess, obesity, diabetes, and increased cardiovascular disease, which threatens to reduce advances in life expectancy. These trends were evident at least 200 years ago, when the descriptions of failing human hearts in obese individuals were recorded: "It is the state in which the heart with very fat people, and even with persons moderately fat, is found oppressed, and suffocated by an enormous mass of fat [46]."

Much progress has been made toward elucidating mechanisms by which obesity increases the risk of heart failure, and specifically, the contribution of free fatty acids (FFAs) [47•, 48•]. A number of recent reviews describing the relationship between circulating fatty acid 
concentrations, their saturation content, and duration of exposure leading either to maladaptive responses in normal hearts [49•] or cardioprotection in failing hearts [50•] have been recently published. These divergent responses underscore the importance of further studies to fully understand the mechanisms linking nutrient excess, and specifically lipid excess, to cellular regulation. We recently summarized proposed mechanisms involved in cardiac lipid utilization and the development of lipotoxicity in the heart that lead to contractile dysfunction [48•]. These mechanisms include altered AMPK signaling, ceramide accumulation, endoplasmic reticulum (ER)-stress, reactive oxygen species (ROS), and mitochondrial dysfunction. This review will provide an update on recent progress in the elucidation of these mechanisms and present additional novel pathways such as autophagy, microRNA, and epigenetics.

\section{Human Myocardial Steatosis and Cardiac Function}

Myocardial steatosis has been used as a biomarker for cardiac lipotoxicity in humans. Advances in magnetic resonance spectroscopy and positron emission tomography [51, 52], have increased our understanding of the pathophysiology of myocardial lipid accumulation and metabolic fate of circulating fatty acids. Analysis of cardiac tissue obtained at the time of surgery has also provided molecular insights into lipid-mediated cellular mechanisms that impair cardiac function in subjects with obesity and T2DM, with or without heart failure $[53 \cdot \bullet, 54,55]$. Of note, changes in cardiac lipid content can also be induced in otherwise healthy individuals subjected to short-term hyperinsulinemia and hyperglycemia [56], or by temporally increasing circulating FFAs in healthy individuals exercising in the fasted state [57]. Conversely, exercise can result in reduced lipid accumulation in obese individuals, leading to improved cardiac function [58•].

Heart failure is associated with increased lipid accumulation in cardiac tissue, which correlates with increased expression of genes for fatty-acid transport proteins such as CD36 [59]. Lipid accumulation in the failing heart may be reversed by mechanical unloading, which partially alleviates mitochondrial dysfunction and insulin resistance [60]. However, reducing circulating FFAs during heart failure is not sufficient to restore cardiac function [61•]. Thus, the accumulation of lipid in normal or diseased hearts reflects complex interactions between the metabolic and neurohumoral milieu and changes in mechanisms governing cardiac lipid uptake and metabolism. Moreover, as will be discussed below, the functional implications of myocardial steatosis are context-dependent, where it may be maladaptive, or could represent adaptations that might be cardioprotective.

\section{Recent Advances in Previously Described Molecular Pathways That Are Implicated in Cardiac Lipotoxicity}

A number of mechanisms that contribute to the development of lipid induced cellular toxicity have been identified. These are summarized in Table 1. Recent advances in our understanding of the contribution of these pathways to the pathophysiology of lipotoxicity in the heart are now summarized.

\footnotetext{
AMP-Dependent Kinase (AMPK) - A Key Metabolic Sensor-Accumulating evidence suggests that AMPK may play a protective role that mitigates cardiac contractile dysfunction in models of diet-induced obesity. AMPK deficiency exacerbates contractile dysfunction and impaired glucose metabolism in mice fed a high-fat diet (HFD) for 20 weeks [62]. Obesity is associated with hypoadiponectinemia. Adiponectin coordinates fatty acid utilization via AMPK-dependent mechanisms that regulate mitochondrial fatty acylCoA import (generation of malonyl-CoA by acetyl-CoA carboxylase), and through induction of CD36 translocation that increases fatty acid uptake [63]. This effect of adiponectin likely increases complete oxidation of fatty acids and limits the likelihood that
} 
toxic lipid intermediates will accumulate. Trimetazidine, an inhibitor of long-chain 3ketoacyl coenzyme A thiolase activates AMPK, reduces lipid accumulation, and restores contractile function in $\mathrm{db} / \mathrm{db}$ diabetic mice [64]. The mechanism of action of trimetazidine also involves amelioration of oxidative stress via increased antioxidant defenses, such as MnSOD. Thus, modulation of AMPK could represent a novel therapeutic approach to mitigate the adverse consequences of myocardial lipotoxicity.

Autophagy- -Self-eating", or autophagy, is a highly conserved process that when disrupted leads to cellular dysfunction. Saturated fatty acids can induce autophagy in endothelial cells via PKC signaling independent of mammalian target of rapamycin (mTOR) [65]. Inhibition of autophagy in endothelial cells ameliorates palmitate-induced cell death [66•]. Studies are required to determine whether similar mechanisms are present in cardiomyocytes.

Ceramide-When fatty acid uptake exceeds oxidative or storage capacity of a tissue, lipids may be shunted into other pathways, such as ceramide, that may alter intracellular signaling pathways and promote apoptotic cell death. Pharmacologic or genetic inhibition of ceramide synthesis improves cardiac structure, function and metabolism in a transgenic mouse model of lipotoxic cardiomyopathy [67•]. Treatment of high-fat fed animals with fenretinide, which inhibits Des1, the rate-limiting enzyme of ceramide biosynthesis, reduced tissue ceramide levels and increased insulin action [68]. The impact of fenretinide on obesityassociated cardiac dysfunction remains to be elucidated. Decreasing ceramide levels using the serine palmitoyl transferase I (SPT I) inhibitor, myriocin, protects against cardiac dysfunction and is associated with increased cardiac glucose utilization [69]. Thus, inhibiting ceramide biosynthesis remains a promising approach for limiting the adverse consequences of myocardial lipotoxicity.

Leptin-Classic studies revealed that leptin may dramatically reverse the adverse effects of lipotoxicity in transgenic models [70•]. Recent studies from our group and others have shown that leptin signaling to the brain is required to reverse steatosis, altered substrate metabolism, mitochondrial dysfunction, and cardiac dysfunction in obese mice with altered leptin signaling [71•, 72]. In addition, leptin replacement, but not caloric restriction, reduced cardiac lipid accumulation in $o b / o b$ mice in part, due to a failure of caloric restriction to rescue defects in PPAR signaling in cardiomyocytes [73].

PPAR Signaling - Lipids are ligands for transcriptional regulators of the PPAR family; thus, regulating FFA delivery significantly impacts PPAR function. In a genetic mouse model of cardiac triglyceride accumulation (adipose triglyceride lipase, $\mathrm{ATGL}^{-/}$), activation of PPARa, but not PPAR $\gamma$, reversed mitochondrial dysfunction and prevented heart failure [74]. Conversely, ATGL-overexpressing mice that exhibit increased triglyceride lipolysis are protected from pressure-overload induced cardiac dysfunction [75] in the absence of any change in diacylglycerol or ceramide levels. Loss of diacylglycerol acyl transferase 1, an important regulator of triglyceride synthesis, reduces PPAR signaling in mice, leading to decreased fatty acid oxidation and ceramide production. These hearts are protected from HFD-induced myocardial dysfunction [76]. Whereas transgenic overexpression of PPAR $\gamma$ increased lipotoxic cardiac injury, deletion of PPARa in these mice resulted in improved cardiac function that was associated with increased FA oxidation and increased triglyceride synthesis [77•]. Thus, PPAR signaling has pleiotropic and context-dependent effects on cardiac substrate utilization and mitochondrial function, which might complicate the utility of modulation of this pathway as a general approach to ameliorating lipotoxic cardiomyopathy. The related transcriptional co-activators PGC-1a and PGC- $1 \beta$ modulate changes in mitochondria volume, oxidative capacity, and the 
coupling of ATP synthesis that occur in response to HFD and diabetes in the $o b / o b$ model of leptin deficiency [78]. Additional studies are required to determine if modulation of PGC1 signaling represents a feasible therapeutic approach for ameliorating the adverse consequences of myocardial lipotoxicity.

p53-In the streptozotocin model of type 1 diabetes (T1D), the progressive decrease in cardiac contractile function was prevented by genetic deletion of $\mathrm{p} 53$, or by reducing OXPHOS complex IV levels via direct suppression of the p53 transcriptional target, cytochrome c oxidase 2 (SCO2). This protection was associated with a decrease in myocardial lipid accumulation and complex IV induction [79]. Interestingly, pressure overload itself can lead to p53-mediated activation of the inflammatory response in adipose tissue, suggesting an interesting cross talk between the heart and peripheral adipose tissue metabolism $[80 \bullet \bullet, 81 \bullet \bullet]$. A role for this pathway in the pathogenesis of lipotoxic cardiomyopathy remains to be elucidated.

Other Signal Transduction Pathways-HFD may induce myocardial insulin resistance by a mechanism involving diacylglycerol activation of PKC signaling and p70S6K activation, which might feedback to inhibit insulin signaling [82]. The resulting defect in insulin-stimulated glucose oxidation was not associated with changes in contractile function, ex vivo or in vivo. Lipids also activate PKC, resulting in decreased $\beta$-adrenergic receptor abundance and reduced cardiac contractile responsiveness to catecholamines [83]. Pharmacologic inhibition of $\beta$-adrenergic receptors with metoprolol in streptozotocin-treated mice inhibited CPT1, but did not prevent cardiac lipid accumulation or oxidative stress. Of note, metoprolol treatment-activated PKB mediated signaling to reduce caspase activation and promote cell survival [84]. Finally, in a recent study in a mouse model of longstanding diet-induced obesity (6 months of $60 \%$ high-fat feeding), cardiac dysfunction was prevented in mice with cardiac-specific deletion of FOXO1, suggesting an important role for nuclear FOXO signaling in mediating obesity and diabetes-related cardiac dysfunction [85•]. Whether or not this pathway plays a role in the early metabolic changes that accompany obesity-induced cardiac dysfunction [86], or is applicable to lower degrees of dietary fat overload, remains to be established.

\section{Novel Molecular Pathways Implicated in Lipotoxicity}

Two novel areas that have recently illuminated our understanding of the interactions between obesity, diabetes, and lipotoxicity are microRNAs (miRs) and epigenetics, which describes the regulation of gene expression through modifications DNA or chromatin.

A number of recent studies have provided interesting insights into the role of microRNAs in the regulation of cardiac and systemic lipid metabolism [87, 88, 81••, 89•], reviewed in [90]. Recent work examining livers of obese mice, found upregulation of miR-143 in the liver as well as in the heart [91••], and genetic reduction of this miR rendered animals resistant to diet-induced obesity and insulin resistance. The role of this microRNA in the pathogenesis of obesity-related cardiac dysfunction remains to be elucidated. Studies examining the relationship between other miRs, insulin resistance, and obesity-related cardiovascular dysfunction remain to be conducted. MicroRNAs are important regulators of lipoprotein metabolism, which play an important role in the delivery of fatty acid substrates to the heart [92]. Interestingly, miR delivery appears to be dependent upon lipoproteins [93•]; thus, it will be of great interest to determine if the altered lipid environment that accompanies obesity and diabetes may regulate the uptake and delivery of miRs to tissues.

Strong evidence supports a role for transgenerational epigenetic mechanisms that may contribute to the current and rapid rise in obesity, diabetes, and its related complications. 
Analysis of the role of epigenetics in the interaction between obesity on cardiac dysfunction is relatively understudied. High-fat diets induce metabolic defects in offspring fed a healthy diet. This is true for both maternal [94] and paternal [95] overnutrition, and may persist for at least three generations in rodents [96]. The adverse effects in the cardiovascular system resulting from parental HFD feeding are seen in otherwise normal offspring fed a healthy diet [97•] and in the absence of obesity [98]. Together, these findings suggest is that high-fat and Western diets may not only have direct cellular lipotoxic effects, but may predispose future generations via epigenetic mechanisms that lead to delayed lipotoxicity and mitochondrial dysfunction that may increase the susceptibility to developing obesity, diabetes and heart disease.

\section{Glucotoxicity Versus Lipotoxicity}

Diets that are enriched for fat and carbohydrate (Western diets) appear to be those that most consistently induce left ventricular dysfunction in rodent models [99•], raising the concept that the total nutrient burden to the heart could accelerate the maladaptation that is observed. For example, an obesogenic western diet impairs Krebs cycle flux and alters gene expression, favoring oxidative stress and ultimately programmed cell death [100]. These studies in animals on a high-fat/high-sucrose "Western diet" underscore the need to account for the contribution of increased generation or flux of glucose-derived metabolites. For example, increased flux via the hexosamine biosynthetic pathway, which increases posttranslational modifications of proteins via $O$-GlcNAcylation has classically been studied in models with increased glucose delivery. This pathway is also increased in conditions associated with increased exposure to fatty acids [101]. Indeed, chronic feeding of Western diets increases $O$-GlcNAc modifications of a number of proteins, including those involved in autophagic flux [102]. In transgenic models, increasing carbohydrate delivery alone might not be deleterious to cardiac function. But these mice with enhanced glucose uptake, and which are protected against cardiac dysfunction in response to hemodynamic challenges, rapidly develop contractile dysfunction when challenged with a HFD [103•]. Similarly, models with a balanced increased myocardial fatty acid utilization and oxidation do not develop left ventricular dysfunction under non-stressed conditions or in response to pressure overload [104•]. Taken together, it is likely that cardiac maladaptation that develops in the context of diet-induced obesity reflects the synergistic interactions of substrate overload and interrelated signaling pathways that are activated by lipid-derived and glucose-derived intermediates.

\section{Cardiac Lipoprotection}

Although there is strong evidence linking diet-induced obesity and lipotoxicity with initiating heart failure, studies in models of heart failure have suggested that increased dietary fat might be cardioprotective. For example, feeding rabbits a diet rich in unsaturated fatty acids prevented pressure or volume overload-induced heart failure [105]. The concept that dietary fatty acid composition or myocardial fat storage may be cardioprotective was recently reviewed [50•]. It has long been appreciated that co-feeding cells with saturated and unsaturated fatty acids can promote lipid droplet accumulation and protection from cell toxicity [106, 107•]. Lipid droplet-binding proteins (perilipins, Plin) may play an important role in the mechanism by which lipid partitioning towards triacylglycerol (TG) storage will reduce lipotoxicity. Specifically, Plin5 antagonizes lipases, thereby preventing ROS damage while maintaining lipid droplet stability. In addition, Plin5, but not Plin1 or Plin2, directly associates with mitochondria and lipid droplets, channeling saturated fatty acids away from oxidation and toward storage [108]. In Plin5 ${ }^{-/}$mice fed normal chow, there is an increase in cardiac fatty acid oxidation, loss of lipid droplet formation and decline in fractional shortening with age [109], supporting a protective role for TG storage to limit the accumulation of toxic lipid intermediates. 
There is also strong evidence that maintaining substrate flexibility and balance is essential in maintaining myocardial function in times of hemodynamic stress, as is the case with ischemic injury. For example, HFD feeding prevented contractile dysfunction following ischemic injury by a mechanism involving maintenance of myofilament expression [110]. In an independent study, similar cardioprotection correlated with altered cardiac mitochondrial substrate utilization through a mechanism associated with inhibition of glucose oxidation via induction of pyruvate dehydrogenase kinase 4 (PDK4) [111]. Likewise, when PDK4 was overexpressed in murine hearts, they were protected from HFD-induced lipid accumulation, likely from enhanced oxidation of fatty acids [112].

Additional mechanisms linking high-fat feeding and cardioprotection in the context of left ventricular remodeling include the potential role of myocardial insulin resistance that develops in the heart when rats are fed a HFD following coronary artery ligation [113•]. Indeed, genetic reduction of insulin signaling limited left ventricular remodeling in the context of pressure overload hypertrophy via a mechanism involving the attenuation of Akt signaling [114•]. High sucrose diet-induced insulin resistance also appears to protect the heart from subsequent bouts of metabolic or hemodynamic challenge [115•]. It should be noted that although acquired insulin resistance is protective in these contexts, complete loss of insulin signaling through genetic ablation of the insulin receptor exacerbates mitochondrial dysfunction and cardiac efficiency in a model of insulin deficient diabetes [116] or pressure overload or post-myocardial infarction remodeling [117, 118].

Induction of the rate-limiting enzyme in the fatty acid desaturation pathway, stearoylcoenzyme A desaturase-1 (SCD1), was observed in rat hearts following three months of a high sucrose diet that was sufficient to induce obesity [119]. Overexpression of SCD1 in primary cardiomyocytes protected cells from numerous lipotoxic effects, including increased ceramide synthesis, reactive oxygen species generation, and apoptosis [119], suggesting that SCD induction is an adaptive mechanism. Finally, increased expression of genes involved in fatty acid synthesis was observed in murine models of lipotoxicity and obesity-related cardiac dysfunction. It remains to be determined if these changes represent an adaptation that attempts to limit lipotoxic injury [120].

\section{Summary, Part 2}

The accumulation of lipids in the heart is associated with cardiac dysfunction in humans and rodents with diet-induced obesity. However, triglycerides per se are not likely to be toxic, but may be a biomarker for the accumulation of more toxic and reactive lipid metabolites. Multiple signaling pathways synergistically interact to impair cardiac structure and function, and emerging mechanisms such as miRNAs and epigenetic regulation will likely have important roles in the pathophysiology of lipotoxicity. The combination of these complex pathways that lead to cardiovascular dysfunction is summarized in Figure 2. The adverse consequences of lipotoxicity are exacerbated to a greater extent by obesogenic diets that promote insulin resistance, and an overall state of nutrient overload. Seemingly paradoxically, increased dietary fat, particularly isocaloric or hypocaloric diets, may lead to cardioprotection in hearts that are already undergoing left ventricular remodeling.

\section{Conclusion}

Although there is clear overlap in the mechanisms by which increased circulating lipids or increased tissue lipid uptake may induce lipotoxicity in vascular and cardiac cells, there is no single unifying mechanism that could be easily manipulated therapeutically to treat or prevent cardiac or vascular disease. This underscores the importance of preventive strategies targeted at delaying the onset of obesity and insulin resistance. The challenge of managing the large numbers of individuals with existing vascular disease remains. Therefore, the 
challenge for the future will be to identify points of convergence in the pathophysiology of cardiovascular disease in obesity that are amenable to therapeutic intervention and that would have the broadest ability to modify clinical outcome. There is no doubt that further characterization of established signaling pathways, such as insulin signaling, inflammation and nitric oxide, may provide a more complete understanding towards these goals. In addition, we anticipate that new advances in emerging fields, such as microRNAs, epigenetics and autophagy, will provide a more complete understanding of how nutrient excess in obesity and diabetes precipitates cellular dysfunction in the heart and vasculature.

\section{Acknowledgments}

A.R. Wende is supported by a National Institutes of Health (NIH) grant K99 HL111322 and a JDRF Advanced Postdoctoral Fellowship 10-2009-672. J.D. Symons is supported by American Heart Association (AHA) Western States Affiliate Grant-In-Aid 06-55222Y, American Diabetes Association Research Grant 7-08-RA-164, and NIH grant R15 HL091493. E.D. Abel is supported by NIH grants R01 DK092065, R01HL108379, U01 HL087947, and is an established investigator of the AHA.

\section{References}

Papers of particular interest have been highlighted as follows:

- Of importance

•• Of outstanding importance

1. Kim JA, Montagnani M, Koh KK, Quon MJ. Reciprocal relationships between insulin resistance and endothelial dysfunction: molecular and pathophysiological mechanisms. Circulation. 2006; 113:1888-1904. [PubMed: 16618833]

2. Jiang ZY, Lin YW, Clemont A, et al. Characterization of selective resistance to insulin signaling in the vasculature of obese Zucker (fa/fa) rats. J Clin Invest. 1999; 104:447-457. [PubMed: 10449437], This manuscript provided some of the first experimental data for pathway specific regulation downstream of vascular insulin resistance. Specifically, decreased activation of IRS-1 and IRS-2 selectively reduces PI3K signaling without altering the MAPK pathway.

3. Rask-Madsen C, Li Q, Freund B, et al. Loss of insulin signaling in vascular endothelial cells accelerates atherosclerosis in apolipoprotein E null mice. Cell Metab. 2010; 11:379-389. [PubMed: 20444418], Evidence is provided that loss of insulin signaling in endothelium increases atherosclerotic lesion formation in part via a mechanism mediated by impaired eNOS activation.

4. Maeno Y, Li Q, Park K, et al. Inhibition of insulin signaling in endothelial cells by protein kinase Cinduced phosphorylation of p85 subunit of phosphatidylinositol 3-kinase (PI3K). J Biol Chem. 2012; 287:4518-4530. [PubMed: 22158866], This study identified a molecular mechanisms by which activation of PKC inhibits IRS1-mediated PI3K activation of eNOS via phosphorylation of the $\mathrm{p} 85$ subunit of PI3K.

5. Geraldes P, King GL. Activation of protein kinase $\mathrm{C}$ isoforms and its impact on diabetic complications. Circ Res. 2010; 106:1319-1331. [PubMed: 20431074]

6. Tang Y, Li G. Chronic exposure to high fatty acids impedes receptor agonist-induced nitric oxide production and increments of cytosolic $\mathrm{Ca}^{2+}$ levels in endothelial cells. J Mol Endocrinol. 2011; 47:315-326. [PubMed: 21994216]

7. Kuboki K, Jiang ZY, Takahara N, et al. Regulation of endothelial constitutive nitric oxide synthase gene expression in endothelial cells and in vivo: A specific vascular action of insulin. Circulation. 2000; 101:676-681. [PubMed: 10673261]

8. Naruse K, Rask-Madsen C, Takahara N, et al. Activation of vascular protein kinase C-beta inhibits Akt-dependent endothelial nitric oxide synthase function in obesity-associated insulin resistance. Diabetes. 2006; 55:691-698. [PubMed: 16505232] , This study provides the first detailed exploration of PKC-activation by obesity-induced insulin resistance and the inhibition of eNOS signaling in vascular tissue. 
9. Abe H, Yamada N, Kamata K, et al. Hypertension, hypertriglyceridemia, and impaired endotheliumdependent vascular relaxation in mice lacking insulin receptor substrate-1. J Clin Invest. 1998; 101:1784-1788. [PubMed: 9541510]

10. Kubota T, Kubota N, Kumagai H, et al. Impaired insulin signaling in endothelial cells reduces insulin-induced glucose uptake by skeletal muscle. Cell Metab. 2011; 13:294-307. [PubMed: 21356519], In addition to further supporting a role of IRS2 in endothelial eNOS signaling, this landmark paper highlights the requirement of intact endothelial cell insulin signaling to regulate myocyte glucose homeostasis.

11. Wheatcroft SB, Shah AM, Li JM, et al. Preserved glucoregulation but attenuation of the vascular actions of insulin in mice heterozygous for knockout of the insulin receptor. Diabetes. 2004; 53:2645-2652. [PubMed: 15448096], This study provides some of the first evidence that impaired insulin signaling may alter NO-mediated regulation of vascular function that may precede defects in insulin mediated muscle glucose utilization.

12. Duncan ER, Walker SJ, Ezzat VA, et al. Accelerated endothelial dysfunction in mild prediabetic insulin resistance: The early role of reactive oxygen species. Am J Physiol Endocrinol Metab. 2007; 293:E1311-E1319. [PubMed: 17711985]

13. Kahn MB, Yuldasheva NY, Cubbon RM, et al. Insulin resistance impairs circulating angiogenic progenitor cell function and delays endothelial regeneration. Diabetes. 2011; 60:1295-1303. [PubMed: 21317296], This study identified an important role for insulin signaling to maintain vascular function by directly regulating cellular repair mechanisms through recruitment of angiogenic progenitor cells.

14. Okamoto H, Nakae J, Kitamura T, et al. Transgenic rescue of insulin receptor-deficient mice. J Clin Invest. 2004; 114:214-223. [PubMed: 15254588]

15. Symons JD, McMillin SL, Riehle C, et al. Contribution of insulin and Akt1 signaling to endothelial nitric oxide synthase in the regulation of endothelial function and blood pressure. Circ Res. 2009; 104:1085-1094. [PubMed: 19342603], The data presented in this study, using both in vitro and in vivo models, found that HFD-induced vascular dysfunction resulted from impaired eNOS phosphorylation independent of significant changes in insulin-mediated activation of Akt, suggested an important role for circulating fatty acids in impairing eNOS function.

16. Abbas A, Imrie H, Viswambharan H, et al. The insulin-like growth factor-1 receptor is a negative regulator of nitric oxide bioavailability and insulin sensitivity in the endothelium. Diabetes. 2011; 60:2169-2178. [PubMed: 21677284], Findings in this report described the cross talk between insulin and IGF-1 receptors in regulating insulin sensitivity and NO action on vascular contractility.

17. Kim F, Pham M, Luttrell I, et al. Toll-like receptor-4 mediates vascular inflammation and insulin resistance in diet-induced obesity. Circ Res. 2007; 100:1589-1596. [PubMed: 17478729] , In an attempt to identify additional mechanisms of diet-induced vascular dysfunction, this study defined a new pathway for TLR4 in mediating saturated fatty acid induced impairment in endothelial NO signaling, inflammation, and insulin resistance.

18. Kim F, Pham M, Maloney E, et al. Vascular inflammation, insulin resistance, and reduced nitric oxide production precede the onset of peripheral insulin resistance. Arterioscler Thromb Vasc Biol. 2008; 28:1982-1988. [PubMed: 18772497]

19. Molnar J, Yu S, Mzhavia N, et al. Diabetes induces endothelial dysfunction but does not increase neointimal formation in high-fat diet fed C57BL/6J mice. Circ Res. 2005; 96:1178-1184. [PubMed: 15879311]

20. Rizzo NO, Maloney E, Pham M, et al. Reduced NO-cGMP signaling contributes to vascular inflammation and insulin resistance induced by high-fat feeding. Arterioscler Thromb Vasc Biol. 2010; 30:758-765. [PubMed: 20093624]

21. Summers SA. Sphingolipids and insulin resistance: The five Ws. Curr Opin Lipidol. 2010; 21:128135. [PubMed: 20216312]

22. Holland WL, Summers SA. Sphingolipids, insulin resistance, and metabolic disease: New insights from in vivo manipulation of sphingolipid metabolism. Endocr Rev. 2008; 29:381-402. [PubMed: 18451260]

23. Zhang QJ, Holland WL, Wilson L, et al. Ceramide mediates vascular dysfunction in diet-induced obesity by PP2A-mediated dephosphorylation of the eNOS-Akt complex. Diabetes. 2012; 
61:1848-1859. [PubMed: 22586587], This study provided strong evidence linking ceramide that is generated by excess fatty acid delivery, to deactivation of the eNOS-Akt pathway in the endothelium. The molecular mechanism is a ceramide-dependent recruitment of the PP2A phosphatase to the eNOS/Akt signaling complex.

24. Wu Y, Song P, Xu J, et al. Activation of protein phosphatase $2 \mathrm{~A}$ by palmitate inhibits AMPactivated protein kinase. J Biol Chem. 2007; 282:9777-9788. [PubMed: 17255104]

25. Knopp RH, Retzlaff B, Walden C, et al. One-year effects of increasingly fat-restricted, carbohydrate-enriched diets on lipoprotein levels in free-living subjects. Proc Soc Exp Biol Med. 2000; 225:191-199. [PubMed: 11082213]

26. Schwartz EA, Reaven PD. Molecular and signaling mechanisms of atherosclerosis in insulin resistance. Endocrinol Metab Clin North Am. 2006; 35:525-549. [PubMed: 16959584]

27. Schwartz EA, Zhang WY, Karnik SK, et al. Nutrient modification of the innate immune response: A novel mechanism by which saturated fatty acids greatly amplify monocyte inflammation. Arterioscler Thromb Vasc Biol. 2010; 30:802-808. [PubMed: 20110572]

28. Kim F, Tysseling KA, Rice J, et al. Free fatty acid impairment of nitric oxide production in endothelial cells is mediated by IKKbeta. Arterioscler Thromb Vasc Biol. 2005; 25:989-994. [PubMed: 15731493], This study provides the first direct link between FFA-induced changes in NO signaling and IKK $\beta$ to IRS- 1 signaling in endothelial cells.

29. Liu K, Zhao W, Gao X, et al. Diosgenin ameliorates palmitate-induced endothelial dysfunction and insulin resistance via blocking IKKbeta and IRS-1 pathways. Atherosclerosis. 2012 In press.

30. Maloney E, Sweet IR, Hockenbery DM, et al. Activation of NF-kappaB by palmitate in endothelial cells: A key role for NADPH oxidase-derived superoxide in response to TLR4 activation. Arterioscler Thromb Vasc Biol. 2009; 29:1370-1375. [PubMed: 19542021]

31. Miriyala S, Gongora Nieto MC, Mingone C, et al. Bone morphogenic protein-4 induces hypertension in mice: Role of noggin, vascular NADPH oxidases, and impaired vasorelaxation. Circulation. 2006; 113:2818-2825. [PubMed: 16769910], Results presented in this manuscript are the first to define BMP4 as a novel regulator of endothelial function through a pathway that requires $\mathrm{p} 47$ phox.

32. Zou MH, Shi C, Cohen RA. Oxidation of the zinc-thiolate complex and uncoupling of endothelial nitric oxide synthase by peroxynitrite. J Clin Invest. 2002; 109:817-826. [PubMed: 11901190]

33. Fonseca FV, Ravi K, Wiseman D, et al. Mass spectroscopy and molecular modeling predict endothelial nitric oxide synthase dimer collapse by hydrogen peroxide through zinc tetrathiolate metal-binding site disruption. DNA Cell Biol. 2010; 29:149-160. [PubMed: 20184449]

34. Wong SW, Kwon MJ, Choi AM, et al. Fatty acids modulate Toll-like receptor 4 activation through regulation of receptor dimerization and recruitment into lipid rafts in a reactive oxygen speciesdependent manner. J Biol Chem. 2009; 284:27384-27392. [PubMed: 19648648]

35. Erridge C, Samani NJ. Saturated fatty acids do not directly stimulate Toll-like receptor signaling. Arterioscler Thromb Vasc Biol. 2009; 29:1944-1949. [PubMed: 19661481]

36. Holland WL, Bikman BT, Wang LP, et al. Lipid-induced insulin resistance mediated by the proinflammatory receptor TLR4 requires saturated fatty acid-induced ceramide biosynthesis in mice. J Clin Invest. 2011; 121:1858-1870. [PubMed: 21490391], This study provided a direct link between inflammatory signaling and the generation of the toxic lipid intermediate ceramide, thereby providing a mechanism for linking inflammation, ceramide, and lipotoxic effects of saturated fatty acids.

37. Takabe K, Paugh SW, Milstien S, Spiegel S. "Inside-out" signaling of sphingosine-1-phosphate: Therapeutic targets. Pharmacol Rev. 2008; 60:181-195. [PubMed: 18552276]

38. Holland WL, Miller RA, Wang ZV, et al. Receptor-mediated activation of ceramidase activity initiates the pleiotropic actions of adiponectin. Nat Med. 2011; 17:55-63. [PubMed: 21186369] , Decreased adiponectin levels often accompany obesity. This study provides the first direct evidence that adiponectin may mediate some of its cardioprotective and beneficial metabolic effects by activating ceramidase activity.

39. Holland WL, Scherer PE. PAQRs. A counteracting force to ceramides? Mol Pharmacol. 2009; 75:740-743. [PubMed: 19158359] 
40. Morales-Ruiz M, Lee MJ, Zollner S, et al. Sphingosine 1-phosphate activates Akt, nitric oxide production, and chemotaxis through a $\mathrm{G}_{i}$ protein/phosphoinositide 3-kinase pathway in endothelial cells. J Biol Chem. 2001; 276:19672-19677. [PubMed: 11278592], This study presents some of the first evidence implicating sphingosine mediated signaling in the regulation of PI3K and Akt signaling to eNOS.

41. Lee S, Zhang H, Chen J, et al. Adiponectin abates diabetes-induced endothelial dysfunction by suppressing oxidative stress, adhesion molecules, and inflammation in type 2 diabetic mice. Am J Physiol Heart Circ Physiol. 2012; 303:H106-H115. [PubMed: 22561304]

42. Pangare M, Makino A. Mitochondrial function in vascular endothelial cell in diabetes. J Smooth Muscle Res. 2012; 48:1-26. [PubMed: 22504486] , A thorough review of recent advances linking changes in mitochondrial ROS production, ion homeostasis, to changes in mitochondrial morphology and dynamics in regulating endothelial function.

43. Shenouda SM, Widlansky ME, Chen K, et al. Altered mitochondrial dynamics contributes to endothelial dysfunction in diabetes mellitus. Circulation. 2011; 124:444-453. [PubMed: 21747057]

44. Makino A, Suarez J, Gawlowski T, et al. Regulation of mitochondrial morphology and function by O-GlcNAcylation in neonatal cardiac myocytes. Am J Physiol Regul Integr Comp Physiol. 2011; 300:R1296-R1302. [PubMed: 21346246]

45. Jheng HF, Tsai PJ, Guo SM, et al. Mitochondrial fission contributes to mitochondrial dysfunction and insulin resistance in skeletal muscle. Mol Cell Biol. 2012; 32:309-319. [PubMed: 22083962]

46. Corvisart des Marets, JN. An essay on the organic diseases and lesions of the heart and great vessels. Vol. 1812. Boston: Bradford \& Read; Of the degeneration of the muscular tissue of the heart into fat; p. 153-156.

47. Abel ED, Litwin SE, Sweeney G. Cardiac remodeling in obesity. Physiol Rev. 2008; 88:389-419. [PubMed: 18391168], An exhaustive and comprehensive review of the mechanisms that lead to obesity-associated cardiac dysfunction in humans and in animal models of obesity.

48. Wende AR, Abel ED. Lipotoxicity in the heart. Biochim Biophys Acta. 2010; 1801:311-319. [PubMed: 19818871], This review provided an update into the molecular mechanisms by which lipid excess impairs cardiac function in humans and animal models.

49. Goldberg IJ, Trent CM, Schulze PC. Lipid metabolism and toxicity in the heart. Cell Metab. 2012; 15:805-812. [PubMed: 22682221] , A recent review with a particular focus on the regulation of delivery of fatty acids to the heart and the role this plays in the development of lipotoxicity.

50. Stanley WC, Dabkowski ER, Ribeiro RF, O'Connell KA. Dietary fat and heart failure: Moving from lipotoxicity to lipoprotection. Circ Res. 2012; 110:764-776. [PubMed: 22383711], This review discusses some of the controversies surrounding lipotoxicity and reviews circumstances in which increased dietary intake of fat may result in cardioprotection.

51. O'Connor RD, Xu J, Ewald GA, et al. Intramyocardial triglyceride quantification by magnetic resonance spectroscopy: In vivo and ex vivo correlation in human subjects. Magn Reson Med. 2011; 65:1234-1238. [PubMed: 21500254]

52. Labbé SM, Grenier-Larouche T, Croteau E, et al. Organ-specific dietary fatty acid uptake in humans using positron emission tomography coupled to computed tomography. Am J Physiol Endocrinol Metab. 2011; 300:E445-E453. [PubMed: 21098737]

53. Niemann B, Chen Y, Teschner M, et al. Obesity induces signs of premature cardiac aging in younger patients: The role of mitochondria. J Am Coll Cardiol. 2011; 57:577-585. [PubMed: 21272749] , A critical examination of cardiac myocytes from human patients identifying how changes in mitochondrial capacity and oxidative coupling are impaired with obesity, linking these defects in mitochondrial function to the increased susceptibility of cardiovascular complications in obese patients.

54. Baranowski M, Blachnio-Zabielska A, Hirnle T, et al. Myocardium of type 2 diabetic and obese patients is characterized by alterations in sphingolipid metabolic enzymes but not by accumulation of ceramide. J Lipid Res. 2010; 51:74-80. [PubMed: 19617631]

55. Marfella R, Di Filippo C, Portoghese M, et al. Myocardial lipid accumulation in patients with pressure-overloaded heart and metabolic syndrome. J Lipid Res. 2009; 50:2314-2323. [PubMed: 19470430] 
56. Winhofer Y, KršKršák M, Janković D, et al. Short-term hyperinsulinemia and hyperglycemia increase myocardial lipid content in normal subjects. Diabetes. 2012; 61:1210-1216. [PubMed: 22396203]

57. Bilet L, van de Weijer T, Hesselink MK, et al. Exercise-induced modulation of cardiac lipid content in healthy lean young men. Basic Res Cardiol. 2011; 106:307-315. [PubMed: 21181177]

58. Schrauwen-Hinderling VB, Hesselink MK, Meex R, et al. Improved ejection fraction after exercise training in obesity is accompanied by reduced cardiac lipid content. J Clin Endocrinol Metab. 2010; 95:1932-1938. [PubMed: 20173015], This study exemplifies how lifestyle interventions can improve cardiac function in obese individuals that correlates with a reduction in cardiac lipid storage.

59. García-Rúa V, Otero MF, Lear PV, et al. Increased expression of fatty-acid and calcium metabolism genes in failing human heart. PLoS One. 2012; 7:e37505. [PubMed: 22701570]

60. Chokshi A, Drosatos K, Cheema FH, et al. Ventricular assist device implantation corrects myocardial lipotoxicity, reverses insulin resistance, and normalizes cardiac metabolism in patients with advanced heart failure. Circulation. 2012; 125:2844-2853. [PubMed: 22586279]

61. Halbirk M, Norrelund H, Moller N, et al. Suppression of circulating free fatty acids with acipimox in chronic heart failure patients changes whole body metabolism but does not affect cardiac function. Am J Physiol Heart Circ Physiol. 2010; 299:H1220-H1225. [PubMed: 20709866] , This study presents a cautionary finding that pharmacological reduction of circulating FFA is not sufficient to improve cardiac contractile function in subjects with heart failure.

62. Turdi S, Kandadi MR, Zhao J, et al. Deficiency in AMP-activated protein kinase exaggerates high fat diet-induced cardiac hypertrophy and contractile dysfunction. J Mol Cell Cardiol. 2011; 50:712-722. [PubMed: 21167835]

63. Fang X, Palanivel R, Cresser J, et al. An APPL1-AMPK signaling axis mediates beneficial metabolic effects of adiponectin in the heart. Am J Physiol Endocrinol Metab. 2010; 299:E721E729. [PubMed: 20739511]

64. Li YJ, Wang PH, Chen C, et al. Improvement of mechanical heart function by trimetazidine in $\mathrm{db} /$ db mice. Acta Pharmacol Sin. 2010; 31:560-569. [PubMed: 20383170]

65. Tan SH, Shui G, Zhou J, et al. Induction of autophagy by palmitic acid via protein kinase cmediated signaling pathway independent of mTOR (mammalian target of rapamycin). J Biol Chem. 2012; 287:14364-14376. [PubMed: 22408252]

66. Khan MJ, Rizwan Alam M, Waldeck-Weiermair M, et al. Inhibition of autophagy rescues palmitic acid-induced necroptosis of endothelial cells. J Biol Chem. 2012; 287:21110-21120. [PubMed: 22556413], This study provides one of the first examples directly linking autophagy to saturated fatty acid induced endothelial cell death.

67. Park T-S, Hu Y, Noh H-L, et al. Ceramide is a cardiotoxin in lipotoxic cardiomyopathy. J Lipid Res. 2008; 49:2101-2112. [PubMed: 18515784], This study described the requirement of ceramide synthesis via serine palmitoyltransferase in the development of cardiac lipotoxicity. Furthermore, it shows that inhibiting this pathway is a potential therapeutic avenue for restoring mitochondrial oxidative function, contractile function, and survival in a mouse model lipotoxic cardiomyopathy.

68. Bikman BT, Guan Y, Shui G, et al. Fenretinide prevents lipid-induced insulin resistance by blocking ceramide biosynthesis. J Biol Chem. 2012; 287:17426-17437. [PubMed: 22474281]

69. Ussher JR, Folmes CD, Keung W, et al. Inhibition of serine palmitoyl transferase 1 reduces cardiac ceramide levels and increases glycolysis rates following diet-induced insulin resistance. PLoS One. 2012; 7:e37703. [PubMed: 22629445]

70. Lee Y, Naseem RH, Duplomb L, et al. Hyperleptinemia prevents lipotoxic cardiomyopathy in acyl CoA synthase transgenic mice. Proc Natl Acad Sci U S A. 2004; 101:13624-13629. [PubMed: 15347805], This study provided the first direct evidence that leptin signaling may protect against the development of lipotoxicity.

71. Sloan C, Tuinei J, Nemetz K, et al. Central leptin signaling is required to normalize myocardial fatty acid oxidation rates in caloric-restricted ob/ob mice. Diabetes. 2011; 60:1424-1434.

[PubMed: 21441440], This manuscript presents direct evidence that intact leptin signaling to the brain is required for systemic changes in obesity induced mitochondrial and cardiac dysfunction. 
72. Keung W, Cadete VJ, Palaniyappan A, et al. Intracerebroventricular leptin administration differentially alters cardiac energy metabolism in mice fed a low-fat and high-fat diet. J Cardiovasc Pharmacol. 2011; 57:103-113. [PubMed: 20980918]

73. Rame JE, Barouch LA, Sack MN, et al. Caloric restriction in leptin deficiency does not correct myocardial steatosis: Failure to normalize PPARa/PGC1a and thermogenic glycerolipid/fatty acid cycling. Physiol Genomics. 2011; 43:726-738. [PubMed: 21427359]

74. Wölkart G, Schrammel A, Dörffel K, et al. Cardiac dysfunction in adipose triglyceride lipase deficiency: Treatment with a PPARa agonist. Br J Pharmacol. 2012; 165:380-389. [PubMed: 21585347]

75. Kienesberger PC, Pulinilkunnil T, Sung MM, et al. Myocardial ATGL overexpression decreases the reliance on fatty acid oxidation and protects against pressure overload-induced cardiac dysfunction. Mol Cell Biol. 2012; 32:740-750. [PubMed: 22158969]

76. Liu L, Yu S, Khan RS, et al. DGAT1 deficiency decreases PPAR expression and does not lead to lipotoxicity in cardiac and skeletal muscle. J Lipid Res. 2011; 52:732-744. [PubMed: 21205704]

77. Son NH, Yu S, Tuinei J, et al. PPAR $\gamma$-induced cardiolipotoxicity in mice is ameliorated by PPARa deficiency despite increases in fatty acid oxidation. J Clin Invest. 2010; 120:3443-3454. [PubMed: 20852389], This study underscored the complex interactions between members of the PPAR family of transcription factors in the regulation of fatty acid uptake, storage and oxidation the heart and showed that lipotoxicity can be ameliorated by simultaneously increasing fatty acid oxidation and triglyceride synthesis.

78. Mitra R, Nogee DP, Zechner JF, et al. The transcriptional coactivators, PGC-1a and beta, cooperate to maintain cardiac mitochondrial function during the early stages of insulin resistance. $\mathrm{J}$ Mol Cell Cardiol. 2012; 52:701-710. [PubMed: 22080103]

79. Nakamura H, Matoba S, Iwai-Kanai E, et al. p53 promotes cardiac dysfunction in diabetic mellitus caused by excessive mitochondrial respiration-mediated reactive oxygen species generation and lipid accumulation / clinical perspective. Circulation: Heart Failure. 2012; 5:106-115. [PubMed: 22075967]

80. Shimizu I, Yoshida Y, Katsuno T, et al. p53-induced adipose tissue inflammation is critically involved in the development of insulin resistance in heart failure. Cell Metab. 2012; 15:51-64. [PubMed: 22225876], Findings in this study revealed important cross talk between adipose and cardiac tissue in response to pressure overload. Specifically, regulation of lipolysis by p53 enhances inflammation and leads to the development of insulin resistance.

81. Grueter CE, van Rooij E, Johnson BA, et al. A cardiac microRNA governs systemic energy homeostasis by regulation of MED13. Cell. 2012; 149:671-683. [PubMed: 22541436], This study revealed that a cardiac-restricted microRNA specifically regulates peripheral metabolic homeostasis. The specific cardiac mediator of this effect remains to be identified.

82. Zhang L, Ussher JR, Oka T, et al. Cardiac diacylglycerol accumulation in high fat-fed mice is associated with impaired insulin-stimulated glucose oxidation. Cardiovasc Res. 2011; 89:148-156. [PubMed: 20729341]

83. Drosatos K, Bharadwaj KG, Lymperopoulos A, et al. Cardiomyocyte lipids impair beta-adrenergic receptor function via PKC activation. Am J Physiol Endocrinol Metab. 2011; 300:E489-E499. [PubMed: 21139071]

84. Sharma V, Sharma A, Saran V, et al. beta-receptor antagonist treatment prevents activation of cell death signaling in the diabetic heart independent of its metabolic actions. Eur J Pharmacol. 2011; 657:117-125. [PubMed: 21296063]

85. Battiprolu PK, Hojayev B, Jiang N, et al. Metabolic stress-induced activation of FOXO1 triggers diabetic cardiomyopathy in mice. J Clin Invest. 2012; 122:1109-1118. [PubMed: 22326951], The data presented in this manuscript directly links HFD induced transcriptional changes via the forkhead transcription factor FOXO1 to cardiac dysfunction and lipotoxic cardiomyopathy in a model of severe diet-induced obesity acting in part via decreased IRS-1 mediated Akt-signaling.

86. Wright JJ, Kim J, Buchanan J, et al. Mechanisms for increased myocardial fatty acid utilization following short-term high-fat feeding. Cardiovasc Res. 2009; 82:351-360. [PubMed: 19147655], This study defines the time course of early changes in myocardial glucose and fatty acid utilization in the evolution of obesity-related cardiac dysfunction. Specifically, the authors identified an early 
defect in GLUT4-mediated glucose utilization that preceded any changes in cardiomyocyte insulin signaling.

87. Ferland-McCollough D, Fernandez-Twinn DS, Cannell IG, et al. Programming of adipose tissue miR-483-3p and GDF-3 expression by maternal diet in type 2 diabetes. Cell Death Differ. 2012; 19:1003-1012. [PubMed: 22223106]

88. Cazanave SC, Mott JL, Elmi NA, et al. A role for miR-296 in the regulation of lipoapoptosis by targeting PUMA. J Lipid Res. 2011; 52:1517-1525. [PubMed: 21633093]

89. Greco S, Fasanaro P, Castelvecchio S, et al. MicroRNA dysregulation in diabetic ischemic heart failure patients. Diabetes. 2012; 61:1633-1641. [PubMed: 22427379] , This study provides one of the first descriptions of altered regulation of microRNAs that relative to non-diabetics with heart failure, may represent a unique molecular signature in hearts of subjects with diabetes and heart failure.

90. Pulakat L, Aroor AR, Gul R, Sowers JR. Cardiac insulin resistance and microRNA modulators. Exp Diabetes Res. 2012 In press.

91. Jordan SD, Kruger M, Willmes DM, et al. Obesity-induced overexpression of miRNA-143 inhibits insulin-stimulated Akt activation and impairs glucose metabolism. Nat Cell Biol. 2011; 13:434446. [PubMed: 21441927], This study identified an essential role for changes in the microRNA (miRNA-143) in the regulation of insulin sensitivity and glucose homeostasis in the liver.

92. Sacco J, Adeli K. MicroRNAs: Emerging roles in lipid and lipoprotein metabolism. Curr Opin Lipidol. 2012; 23:220-225. [PubMed: 22488426]

93. Vickers KC, Palmisano BT, Shoucri BM, et al. MicroRNAs are transported in plasma and delivered to recipient cells by high-density lipoproteins. Nat Cell Biol. 2011; 13:423-433. [PubMed: 21423178], This novel study provided direct evidence that high density lipoproteins (HDL), may serve as carriers of endogenous microRNAs, which in turn will regulate function in remote target organs.

94. Alfaradhi MZ, Ozanne SE. Developmental programming in response to maternal overnutrition. Front Genet. 2011; 2:1-12. [PubMed: 22303300]

95. Ng S-F, Lin RCY, Laybutt DR, et al. Chronic high-fat diet in fathers programs $\beta$-cell dysfunction in female rat offspring. Nature. 2010; 467:963-966. [PubMed: 20962845]

96. Dunn GA, Bale TL. Maternal high-fat diet effects on third-generation female body size via the paternal lineage. Endocrinology. 2011; 152:2228-2236. [PubMed: 21447631]

97. Carone BR, Fauquier L, Habib N, et al. Paternally induced transgenerational environmental reprogramming of metabolic gene expression in mammals. Cell. 2010; 143:1084-1096. [PubMed: 21183072], This manuscript provides strong evidence that environmental factors, including dietary intake, have transgenerational effects on gene expression involved in metabolic function.

98. Rudyk O, Makra P, Jansen E, et al. Increased cardiovascular reactivity to acute stress and saltloading in adult male offspring of fat fed non-obese rats. PLoS One. 2011; 6:e25250. [PubMed: 22043281]

99. Wilson CR, Tran MK, Salazar KL, et al. Western diet, but not high fat diet, causes derangements of fatty acid metabolism and contractile dysfunction in the heart of Wistar rats. Biochem J. 2007; 406:457-467. [PubMed: 17550347], This study was among the first to describe differential effects of increased dietary fat, versus the combination of increased dietary fat and carbohydrate in precipitating left ventricular dysfunction.

100. Ballal K, Wilson CR, Harmancey R, Taegtmeyer H. Obesogenic high fat western diet induces oxidative stress and apoptosis in rat heart. Mol Cell Biochem. 2010; 344:221-230. [PubMed: 20676734]

101. Cooksey RC, McClain DA. Increased hexosamine pathway flux and high fat feeding are not additive in inducing insulin resistance: Evidence for a shared pathway. Amino Acids. 2011; 40:841-846. [PubMed: 20658157]

102. Marsh SA, Powell PC, Dell'italia LJ, Chatham JC. Cardiac $O$-GlcNAcylation blunts autophagic signaling in the diabetic heart. Life Sci 2012. In press.

103. Yan J, Young ME, Cui L, et al. Increased glucose uptake and oxidation in mouse hearts prevent high fatty acid oxidation but cause cardiac dysfunction in diet-induced obesity. Circulation. 2009; 
119:2818-2828. [PubMed: 19451348], This study provided direct evidence of the adverse consequences to the myocardium of forced increase in utilization of glucose and fatty acids.

104. Kolwicz SC, Olson DP, Marney LC, et al. Cardiac-specific deletion of acetyl CoA carboxylase 2 (ACC2) prevents metabolic remodeling during pressure-overload hypertrophy. Circ Res. 2012; 111:728-738. [PubMed: 22730442] . This study describes a transgenic model with a lifelong increase in myocardial fatty acid oxidation that does not develop any evidence of lipotoxic compromise and exhibits preserved function when challenged with pressure overload, underscoring that complete fatty acid oxidation is not intrinsically damaging to the heart even when it is occurring at high levels.

105. Den Ruijter HM, Verkerk AO, Schumacher CA, et al. A diet rich in unsaturated fatty acids prevents progression toward heart failure in a rabbit model of pressure and volume overload. Circulation: Heart Failure. 2012; 5:376-384. [PubMed: 22474247]

106. Gordon GB. Saturated free fatty acid toxicity : II. Lipid accumulation, ultrastructural alterations, and toxicity in mammalian cells in culture. Exp Mol Pathol. 1977; 27:262-276. [PubMed: 561704]

107. Greenberg AS, Coleman RA, Kraemer FB, et al. The role of lipid droplets in metabolic disease in rodents and humans. J Clin Invest. 2011; 121:2102-2110. [PubMed: 21633178], This review presents a focused assessment of the role that lipid droplets play in regulating physiology and metabolism in the development of diabetic complications with a focus on liver, muscle, and adipocytes.

108. Wang H, Sreenevasan U, Hu H, et al. Perilipin 5, a lipid droplet-associated protein, provides physical and metabolic linkage to mitochondria. J Lipid Res. 2011; 52:2159-2168. [PubMed: 21885430]

109. Kuramoto K, Okamura T, Yamaguchi T, et al. Perilipin 5, a lipid droplet-binding protein, protects heart from oxidative burden by sequestering fatty acid from excessive oxidation. J Biol Chem. 2012; 287:23852-23863. [PubMed: 22532565]

110. Cheng Y, Li W, McElfresh TA, et al. Changes in myofilament proteins, but not $\mathrm{Ca}^{2+}$ regulation, are associated with a high-fat diet-induced improvement in contractile function in heart failure. Am J Physiol Heart Circ Physiol. 2011; 301:H1438-H1446. [PubMed: 21765056]

111. Berthiaume JM, Young ME, Chen X, et al. Normalizing the metabolic phenotype after myocardial infarction: Impact of subchronic high fat feeding. J Mol Cell Cardiol. 2012; 53:125133. [PubMed: 22542451]

112. Chambers KT, Leone TC, Sambandam N, et al. Chronic inhibition of pyruvate dehydrogenase in heart triggers an adaptive metabolic response. J Biol Chem. 2011; 286:11155-11162. [PubMed: 21321124]

113. Christopher BA, Huang HM, Berthiaume JM, et al. Myocardial insulin resistance induced by high fat feeding in heart failure is associated with preserved contractile function. Am J Physiol Heart Circ Physiol. 2010; 299:H1917-H1927. [PubMed: 20852054], The studies presented in this manuscript focus on the role that HFD-feeding may play in protecting against pathological insults by inducing insulin resistance in the cardiomyocyte.

114. Shimizu I, Minamino T, Toko H, et al. Excessive cardiac insulin signaling exacerbates systolic dysfunction induced by pressure overload in rodents. J Clin Invest. 2010; 120:1506-1514. [PubMed: 20407209], This study provides direct evidence that pressure overload and myocyte stretch increases insulin signaling in the heart and this increased insulin signaling may contribute to adverse left ventricular remodeling by hyperactivating Akt. The study provides a mechanism for potential beneficial effects of myocardial insulin resistance.

115. Harmancey R, Lam TN, Lubrano GM, et al. Insulin resistance improves metabolic and contractile efficiency in stressed rat heart. FASEB J. 2012; 26:3118-3126. [PubMed: 22611083], This report suggests that insulin resistance resulting from high sucrose feeding may be a mechanism that protected rat hearts from excess fuel delivery.

116. Bugger H, Riehle C, Jaishy B, et al. Genetic loss of insulin receptors worsens cardiac efficiency in diabetes. J Mol Cell Cardiol. 2012; 52:1019-1026. [PubMed: 22342406]

117. Hu P, Zhang D, Swenson L, et al. Minimally invasive aortic banding in mice: Effects of altered cardiomyocyte insulin signaling during pressure overload. American Journal of Physiology Heart and Circulatory Physiology. 2003; 285:H1261-H1269. [PubMed: 12738623] 
118. Sena $\mathrm{S}, \mathrm{Hu} \mathrm{P}$, Zhang D, et al. Impaired insulin signaling accelerates cardiac mitochondrial dysfunction after myocardial infarction. J Mol Cell Cardiol. 2009; 46:910-918. [PubMed: 19249310]

119. Matsui H, Yokoyama T, Sekiguchi K, et al. Stearoyl-CoA desaturase-1 (SCD1) augments saturated fatty acid-induced lipid accumulation and inhibits apoptosis in cardiac myocytes. PLoS One. 2012; 7:e33283. [PubMed: 22413010]

120. Ge F, Hu C, Hyodo E, et al. Cardiomyocyte triglyceride accumulation and reduced ventricular function in mice with obesity reflect increased long chain fatty acid uptake and de novo fatty acid synthesis. J Obes. 2012; 2012:205648. [PubMed: 22132320] 


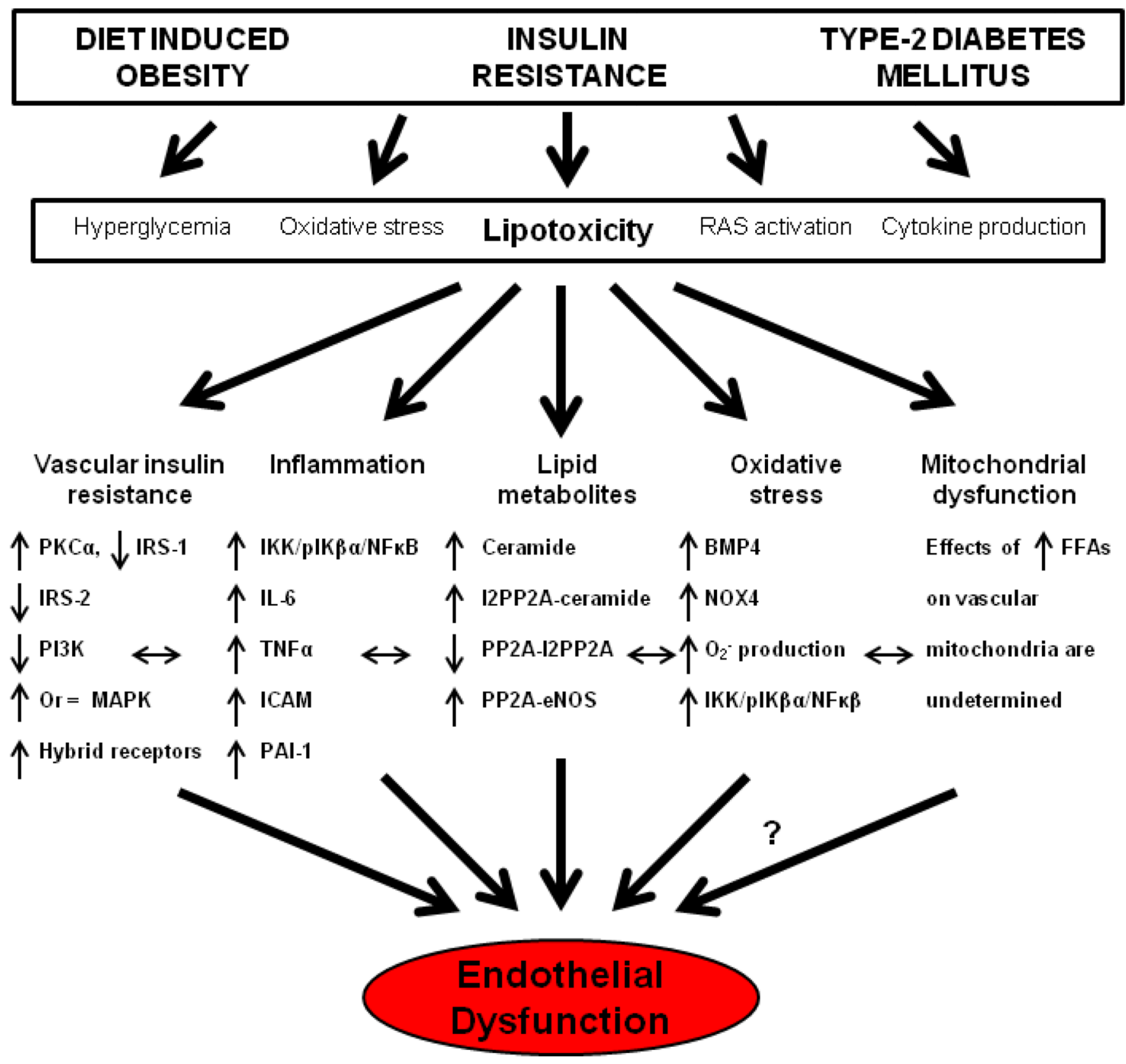

Fig. 1.

Multiple mechanisms contribute to endothelial dysfunction that exists in subjects with dietinduced obesity, insulin resistance, and type 2 diabetes mellitus. Of these, lipotoxicity can decrease endothelial function by: (i) impairing agonist-induced signaling to eNOS in endothelial cells and blood vessels; (ii) increasing inflammation; (iii) stimulating accrual of toxic sphingolipids e.g., ceramide; (iv) promoting oxidative stress to an extent that overwhelms the antioxidant environment; and/or by (v) potentially precipitating mitochondrial dysfunction. Cross talk between these pathways is likely. Definition of abbreviations: FFA—Free fatty acids; $\mathrm{PKCa}$-Protein kinase $\mathrm{C}$ alpha; IRS-1—Insulin receptor substrate-1; IRS-2-Insulin receptor substrate-2; PI3K-Phosphoinositide 3kinase; MAPK - Mitogen activated protein kinase; IKK-Inhibitor of kappa B kinase; $\mathrm{p}$ phosphorylated; NFкB-Nuclear factor kappa-beta; IL-6_-Interleukin-6; TNFa-Tumor 
necrosis factor-alpha; ICAM-Intercellular adhesion molecule; PAI-1-Plasminogen activator inhibitor-1; I2PP2A-Inhibitor 2 of protein phosphatase 2A; PP2A—Protein phosphatase 2A; eNOS-Endothelial nitric oxide synthase; BMP4-Bone morphogenetic protein 4; NOX4-NADPH oxidase 4. For lipid metabolites, the arrows represent the interactions between ceramide, I2PP2A, PP2A, and eNOS 

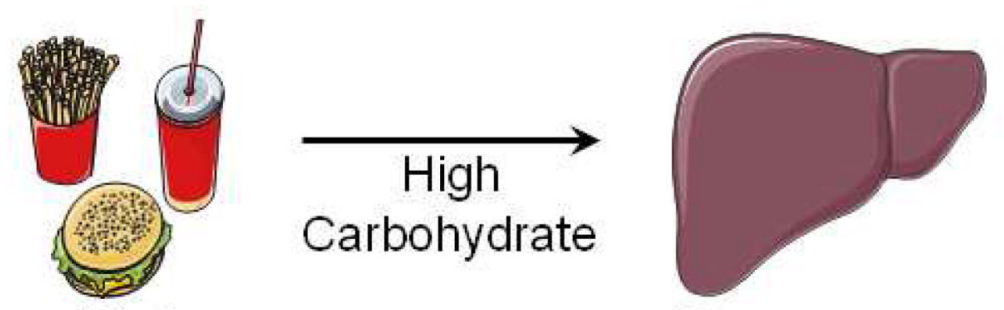

1 Dietary

De novo

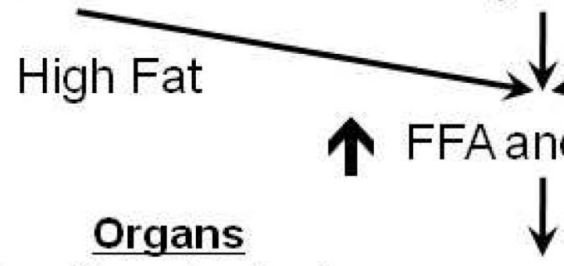

Cardiac steatosis

Vascular dysfunction
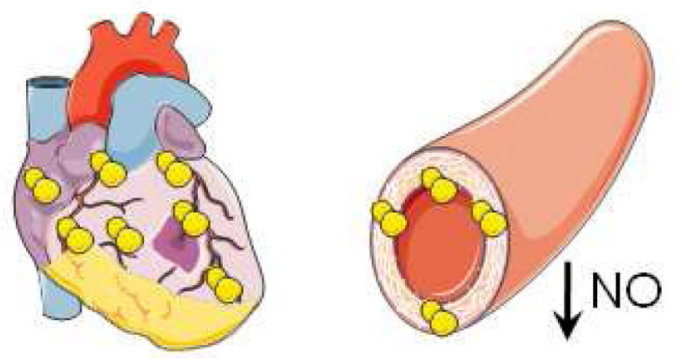

Pathway Regulation by Lipids

$\downarrow$ Adipo
$\downarrow$ Akt
$\downarrow$ AMPK
$\uparrow$ Autophagy
$\uparrow$ Ceramide
$\downarrow$ IGF-1
$\uparrow$ IKK
$\uparrow$ IL-6

$\downarrow$ IRS $1 / 2$

$\uparrow \mathrm{p} 53$

$\downarrow$ Leptin

$\uparrow \mathrm{PKC}$

$\uparrow \downarrow$ miR

$\downarrow$ eNOS

$\uparrow$ PP2A

$\uparrow \downarrow$ PPAR

$\downarrow$ Plin

$\uparrow$ NOX

$\uparrow N F-\kappa \beta$

$\uparrow$ ROS

$\uparrow$ TLR4

$\uparrow$ O-GlcNAc

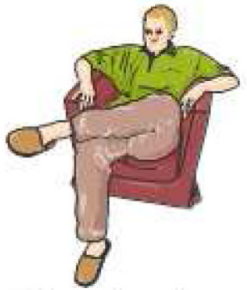

Physical

Expenditure

\section{thesis}

Fig. 2.

Schematic representation of pathways and mechanisms of lipotoxicity. Nutrient excess, from diet, increased de novo synthesis of lipids, and reduced energy expenditure, eventually overwhelms storage and oxidation. This leads to lipid accumulation that will impair cardiac contractile function, nitric oxide signaling in the vasculature, and the function of cellular organelles. Changes in intracellular signaling result in ER stress, mitochondrial dysfunction and reactive oxygen species generation, as well as persistent modifications of DNA and histones. The overall result is dysregulation of various pathways (discussed in the text) that have far reaching consequences. Figure was produced using Servier Medical Art (www.servier.com) 


\section{Table 1}

Proposed mechanisms of lipid-induced regulation of cardiovascular cellular function

\begin{tabular}{|c|c|}
\hline Mechanism & Observation \\
\hline AMPK & Activation protects against FA-induced cell death [62-64] \\
\hline \multirow[t]{3}{*}{ Autophagy } & Enhanced by FA-induced activation of PKC [65] \\
\hline & Enhanced by WD via $O$-GlcNAcylation of autophagy proteins [102] \\
\hline & Inhibition protects against FA-induced cell death in endothelium [66] \\
\hline \multirow[t]{2}{*}{ Ceramide } & Enhances PP2A-eNOS mediated endothelial dysfunction [23] \\
\hline & Inhibition protects against FA-induced cell death [67-69] \\
\hline $\begin{array}{l}\text { Endoplasmic reticulum (ER) } \\
\text { stress }\end{array}$ & Enhanced by FA-induced activation of $\mathrm{Ca}^{2+}$-mediated activation of BCL-2, BAX, and BAK [48] \\
\hline Leptin & $\begin{array}{l}\text { Treatment protects against cardiac dysfunction by reducing lipid accumulation and restoring PPAR signaling } \\
\text { [73] }\end{array}$ \\
\hline \multirow[t]{2}{*}{ Lipid (TAG) storage } & $\begin{array}{l}\text { Feeding unsaturated FA, protects against saturated FA-induced cell death by sequestering lipids in } \\
\text { intracellular droplets }[106,107]\end{array}$ \\
\hline & Perlipins regulate FA-storage to regulate and maintain normal oxidative balance [108, 109] \\
\hline MicroRNA & FA-induced changes in miR regulation results in insulin resistance [91] \\
\hline Mitochondrial function & FA-induced mitochondrial fragmentation and increased reactive oxygen species (ROS) [45] \\
\hline
\end{tabular}

FA: fatty acid; TAG: triacylglycerol; WD: western diet 